\title{
Supporting Data-Driven Decision Making in a Canadian School District
}

\author{
Stephanie Pagan, Katherine Magner, Christine Thibedeau \\ Ottawa-Carleton District School Board
}

\begin{abstract}
A main objective in educational settings is to build system and school capacity in data driven decisionmaking and evidence-informed practice in support of the development, monitoring, and measuring of initiatives targeted to support student achievement and well-being. In 2016-2017, the Ottawa-Carleton District School Board (OCDSB) implemented an innovative Data Support Model to provide support to senior staff, school administrators and teams of educators: (1) in the exploration/understanding of data reflecting student achievement/attitudes/ demographics, (2) in the design of school-specific initiatives, and (3) through the development of online and interactive data resource tools. This paper discusses the conceptual framework and collaborative approach of the model, as well as the use and impact of the model. The results indicate a strong positive relation between the Data Support Model and improved Data Literacy at both a systemand school-level. Implications and opportunities for model improvement and growth are discussed. There has been considerable commitment from participants and a continued desire to grow and enhance this model. With time, it is anticipated that student outcomes will also be positively impacted. This approach is unique to this school district and as such, can serve as a valuable model to other school districts.
\end{abstract}

\section{Introduction}

The effective use of data for decision-making and evidence-informed practice is a growing expectation in schools [1], [6] intended to help identify student needs, design targeted school improvements, and implement focused supports and instructional improvement. Arising from this expectation, is the understanding that all educators should possess some degree of data literacy skills that would afford them the capability to understand, interpret, and use data effectively to inform decisions [7], [6]. Educators have access to a range of data sources (e.g., student grades, standardized assessments, summative and formative assessments, student work, observations, etc.) but may lack the adequate skills, time, or supports to fully explore the data and use it to effect change. The term Data Literacy is broadly defined as the ability to read, understand, create and communicate data as information [5] and when used appropriately, is a skill that positively impacts educator instructional practices [2], [10], improves understanding of student progress [4], and affects student achievement [8]. Thus, building both system- and school capacity in the effective use of data is necessary to build the opportunity for insight and action planning.

Research has presented particular conditions that help facilitate capacity-building for data literacy and the increased use of data in schools. These conditions include: (1) encouraging collaboration among educators, (2) building common goals/purposes for data use, (3) using multiple data elements or triangulation of data points related to educator's own context, and (4) providing sufficient time or embedding data use in current work contexts [11], [1]. Furthermore, utilizing a model that incorporates elements of direct coaching and responsive support is beneficial and effective in promoting data literacy growth [10], [1]. Educator needs for support for data use are also well documented and include such forms as worksheets, portfolios, and feedback [11]. Moreover, the provision of tools has been shown to have positive reception from educators. Technological tools (e.g., data spreadsheets, rubrics, protocols) in particular, have been shown to support learner's data management, analysis, and interpretation capabilities [10]. Similarly, computer data systems that provide educators with data in an efficient and user-friendly fashion have been shown to be supportive in educator use of data [12].

Overall, building capacity in data literacy should be viewed as a learning process. This process is best described by Marsh and Farrell [7] as one where "individuals make sense of information and construct new knowledge through activity and social interactions, mediated by prior knowledge, beliefs, and experiences" (p. 274). It is also important to keep in mind that data literacy skills take time to develop and using a long-term collaborative approach where instruction is communicated explicitly and concretely, will encourage the most desired results [1].

With the goals of improved data literacy and capacity-building in mind, the OCDSB introduced the Data Support Model, a supportive model promoting the development of Data Literacy skills as well as a culture of data use in our schools. This paper will present the benefits and impact of the innovative Data Support Model currently being used in the OCDSB. 
As such, the goals of this paper are to discuss the following:

1. What does the Data Support Model look like?

2. How is the Use of the Data Support Model being used?

3. What is the Impact of the Data Support Model?

4. What Opportunities for Growth have been identified? What are the Next Steps?

\section{Context}

There are 76 public school boards in Ontario, Canada; the Ottawa-Carleton District School Board (OCDSB) is the largest in Eastern Ontario. The OCDSB serves approximately 75,000 students across 143 schools (113 elementary and 30 secondary sites). Spanning an area of 2,780 square kilometres, schools are grouped according to their geographic location into one of six superintendencies each led by a Superintendent of Instruction.

The OCDSB has an internal research division called The Research, Evaluation and Analytics Division (READ). This division is both responsible for and a collaborator in a variety of research-based projects and initiatives. As part of its core mandate, READ is responsible for building capacity in the effective use of data to support implementation and monitoring of system initiatives.

\section{Conceptual Framework}

\subsection{What does the Data Support Model look like?}

Historical Background. The research division at the OCDSB has long supported building system capacity in Data Literacy - the ability to read, understand, create and communicate data as information [5]. Historically, data support has been provided through half-day (3-hour) working sessions with available school teams (e.g., school administrators and 2 to 3 staff) to reflect on multiple sources of data as part of the professional learning cycle associated with the development of School Learning Plans (SLPs). These annual workshops generally took place at the beginning of the school year with additional support occuring on an as-needed basis. In July 2016, a cross-departmental debriefing session reviewing the Data Support Model, identified areas of strength and potential areas for improvement moving forward. Focused areas needing further consideration included:

- The sheer amount/volume of data available to schools was overwhelming for administrators. The decision of what data source to use and for what purpose was a difficult one.
- The existing electronic reporting tools available to administrators were complex, cumbersome, unreliable, and caused frustration. Schools typically only accessed these tools once or twice a year and it was often during the short working sessions provided at the beginning of the year.

- Staff in the research division felt disconnected from the SLP process. Typically, the only point of contact research staff had with schools was during the short working sessions.

Based on this discussion, it became clear that to build greater system capacity in data literacy, a revision to the Data Support Model would be needed.

The Revised Model. In 2016-2017, the OCDSB embarked on a new vision for providing active data support whereby a Data Support Lead (DSL) from the District's research division was partnered with a Superintendent of Instruction to support the group of schools within their Superintendency. A main objective of this revised model was to build greater capacity at the system- and school-level in supporting data-driven decision making and evidence informed practice in the development, monitoring, and measuring of goals outlined in School Learning Plans.

Using available data sources, Data Support Leads (DSLs) assist senior staff, school administrators and teams of educators in exploring/understanding school and district data reflecting student performance/ attitudes/demographics.

This encourages the use of data and evidencebased findings in the execution of the School Learning Plan. Over the course of a schoolyear, individual schools are responsible for developing a School Learning Plan (SLP) that sets goals for improvement and establishes timelines for when goals will be achieved by moving through four phases: Plan, Act, Observe, and Reflect.

DSLs also provide data guidance and support in the design of school-specific initiatives when there is a desire to capture information or monitor impact of such initiatives. This collaborative and organic approach contributes to growth in personal Data Literacy as well as to the transfer of knowledge from one project to another. The sharing of this knowledge as well as the work being done with the DSLs frequently occurs spontaneously in large group contributions at Superintendency meetings throughout the school year and in smaller sessions at the request of school administrators.

In addition to providing active data support, DSLs meet frequently to discuss, plan, and create various data related resources. An online Data Support Toolkit is available to Superintendents and school administrators and its contents are designed to serve the diverse needs and variability in individual comfort and expertise with Data Literacy. The Data Support Toolkit provides users with the following tools and resources: 
- A welcome page to the Data Support Toolkit that provides information about the available resources with embedded links for quick access. Additionally, Board level reports on various topics are also provided;

- A reference guide indicating available data sources. This document includes descriptions of the data sources, directions for how and where to access them, and the name of a contact person who can provide additional assistance if needed;

- Data spreadsheets from multiple sources that provide year to year results at both a school and Board level. Each data spreadsheet comes with a companion document that describes the data resource, how to interpret the data, and provides guiding questions for consideration when interpreting the information;

- Activities connected to specific data resources that school administrators can choose to use with their school staff when examining their school data;

- Short video modules that provide guiding instructions for interpreting school data for particular data sources;

- A variety of Tools and Tipsheets that serve to provide guided instruction and information related to various Data Literacy topics. Some examples include:

○ Creating Surveys: Survey Writing Basics, Writing Good Questions

○ Creating Student Profiles

○ Monitoring SLPs

- How to Identify Marker Students

- Collecting, Analyzing, and Using Qualitative Data

○ How to Improve Parent Engagement

○ How to Conduct Focus Groups

These tools contribute to individual learning and further build system capacity for Data Literacy.

This revised Data Support Model relies on a number of key components to promote effective capacity building. First, communication is a critical feature whereby Data Support Leads (DSLs) have regular meetings and communications with their Superintendent of Instruction (SOI) and encourage open communication with school administrators on a regular basis. Furthermore, DSLs frequently meet as a team to discuss approaches and provide updates of support being provided in their own superintendency. This results in open discussions about what works and does not work in the model, and also serves to mitigate differences in data support provided across superintendencies in an attempt to provide consistency across the system.

Secondly, system and school level awareness and buy-in to the Data Support Model has required strategic planning for increasing the visibility of Data Support Leads (DSL) at these levels. Over the course of the school year, DSLs attend, contribute information to, and support/present at five
Superintendency meetings. These meetings are structured with specific goals in mind and are led by their respective Superintendent. All school administrators are in-attendance, as are representatives from other District departments. These face-to-face interactions are critical to promoting and increasing awareness of the Data Support Model.

Thirdly, the Data Support Model utilizes a targeted approach. In collaboration with their Superintendent of Instruction (SOI), Data Support Leads (DSLs) consider various data sources to help identify schools who could benefit from receiving more individualized support in moving through the phases of their SLP development. As a team, the SOI and DSL develop an actionable plan to provide school teams with targeted support.

Next, Data Support Leads provide support in a number of data related areas. The types of support most commonly sought include: identifying available data resources, selection of appropriate data sources for given projects, examination/interpretation of data, design/implementation of various data collection initiatives (e.g., surveys, focus groups), conducting larger scale projects, creating reports, monitoring/measuring impact, dissemination of information and providing resources/tools.

Finally, Data Support Leads deliver data support in a variety of ways through individual school visits, conference calls, Google hangout, online resources, and in large group settings at Super intendency meetings.

\section{Methodology}

To learn more about the effectiveness, challenges, and opportunities related to the Data Support Model and its components, formal and informal district feedback has been gathered on the DSM since the implementation of the model in 2016. On two occasions (2016-2017 and 2018-2019), reflections and feedback regarding the DSM have been collected from school administrators using self-report surveys. At various points throughout the school-year, the Data Support Lead also has the opportunity to directly connect with school administrators and their Superintendent of Instruction. Additionally, anecdotal evidence has been collected that speaks to the impact of the Data Support Model and suggestions for change and continued growth. Lastly, collection of individual "voice" was conducted and is a powerful indicator of participant experience with the Data Support Model.

\section{Analysis}

\subsection{Use and Impact of the Data Support Model}


Use of the Data Support Model. Since implementation, this model has continued to evolve and grow. As of Spring 2019, the majority of schools $(65 \%)$ are currently collaborating with their Data Support Lead to explore available sources of data, interpret data, and use data to inform practice. This number, however, varies across Superintendencies as evidenced by a range of $20 \%$ to $96 \%$. Communication with DSLs has also become more frequent since the implementation of the revised Data Support Model and there is an indication of increased comfort and awareness in requesting data support. School administrators are seeking support in a variety of ways, with contact at Superintendency meetings being most popular (82\%), followed by e-mail correspondence $(76 \%)$, school visits $(59 \%)$, and telephone/conference calls $(52 \%)$. Examination of when school administrators are seeking support indicates a clear pattern associated with School Learning Plan (SLP) deliverables. This means that the greatest data support needs tend to be expressed at the beginning of the school year (i.e., September/October) and gradually decrease as the school year progresses (see Figure 1). Once again, however, the frequency with which school administrators collaborate with their DSL varies according to Superintendency. For instance, a more involved Superintendency will see a frequency of collaboration 1.5 times higher than the overall frequency.

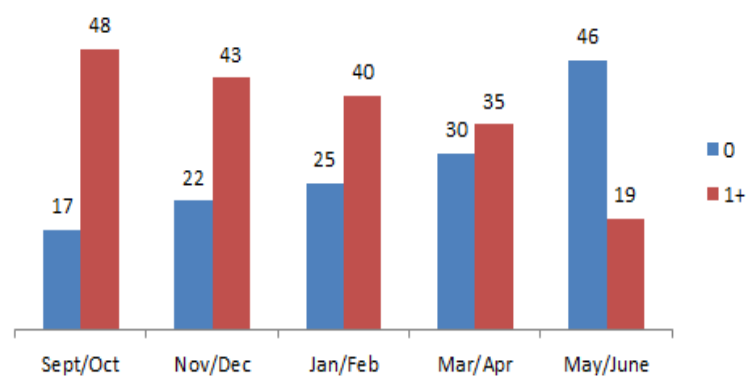

Figure 1. School Administrator. Reported frequency

of collaboration with DSL during the school year School administrators also reported on the frequency with which they access the Data Support Toolkit. Overall, 62\% reported accessing the online Data Support Toolkit. Not surprisingly, the majority (70\%) of school administrators who reportedly collaborate with their DSLs also report accessing the Toolkit. Reasons for accessing the Data Support Toolkit included: seeking additional resources/data support (79\%), for assistance with SLP submissions/timelines (71\%), seeking materials to use at Professional Development (PD) days and/or at staff meetings (59\%), and searching availability/release of new data (59\%). School administrators were also asked to indicate how useful they felt various components of the Data Support Toolkit are. For all components (i.e., data spreadsheets, activities, tipsheets) most (50-57\%) reported they were either somewhat or very useful. Interestingly across each component, at least $10 \%$ of respondents who have accessed the toolkit, reported not having used particular resources.

Impact of the Data Support Model. School administrators were asked to indicate how valuable they felt the Data Support Model is to the District and to individual schools. An overwhelming majority (98\%) reported that the Data Support Model is somewhat or very valuable. Additionally, there is an indication of impact occurring at each level (i.e., system and school) of the Data Support Model. Collection and reflection on individual voice, observations, conversations, and anecdotal evidence substantiates improved capacity building and knowledge growth as a result of the revised Data Support Model. Data Support Leads have expressed seeing staff improvements in understanding the various data resources and the capacity for administrators to answer targeted questions.

"I have noticed increased capacity among the schools I have worked with. I am also seeing the model evolve beyond basic data exploration -- there is greater buy-in to the value of having this expertise in the District."

Further, school administrators reported that the Data Support Model:

“...helps in giving direction for implementing new strategies to help improve student learning."

"...has served as a good reference and a guide for my own learning and as I prepare learning opportunities for staff."

"...has provided support in the area of data literacy for all administrators and school Staff."

“...has given me great insight on my student community. It has helped me know where we are doing well and where we need to improve. It has also helped me determine specific groups to target in terms of growth and achievement."

The Data Support Model has also positively impacted staff confidence in using, sharing, and explaining to others the nature and use of data for specific goals. School administrators shared:

"It has given me a foundation for my observations and has helped me to explain to 
staff why we need to make certain intentional moves to support students."

"Data analysis has not always been a strength. I feel supported by the model."

"My DSL is very knowledgeable and supportive. It has given me more confidence in some of my decisions, next steps as they are based on feedback and data, especially District data."

Data Support Leads further corroborate the feelings of school administrators by saying:

"School administrators have been very candid and open about their insecurities working with data -- knowing they have support I think is positive and they are becoming comfortable asking for assistance; For those who have greater data skill -- I find they are moving to more complex needs and now know where to find it."

Further impact of the model is seen in the transfer of skills, experiences, and support to new school initiatives and goals; as well as to different schools with similar goals. School administrators and Superintendents shared:

"It provides the forum to have discussions with staff about how we can use data to inform instruction." "Has allowed us to make decisions and set directions based on data, in some cases making goals measurable."

"I think that it has resulted in me asking better questions of schools in my superintendency, particularly related to their school learning plans. This is because the data was in a much more accessible format, and there was a "data miner" -- support lead, available to assist me and my schools. It made data less scary and more helpful."

Finally, the continued relationship-building and increased collaborative opportunities related to school initiatives and groups of schools has been significantly impacted by this revised Data Support Model.

"Having a contact person as well as easily accessible resources has helped me quickly access information/have my questions answered."

"The DSM has assisted me in focusing my time (the data is at my fingertips). I also have an incredible support in my DSL who has assisted me in better understanding the data and evidence."

"Working with my DSL has been awesome! She is knowledgeable and can give us ideas about how best to gather data. She also keeps us to our timelines. This is vital. I fear that if I was doing it within the school only, our data collection would fall off."

"Having had the opportunity to meet with and work with my DSL has increased my comfort to reach out to ask questions. I don't feel judged for potentially asking "dumb" questions, even if I have asked them several times over the past several years. This relationship is critical from my point of view."

Overall, taken together the individual voice, observations, conversations, and anecdotal evidence presented above support a strong positive relation between the Data Support Model and improved Data Literacy at both a system- and school-level. There has been considerable buy-in at both the system- and school-level as well as a continued desire to grow and enhance this collaborative model.

\section{Discussion}

This collaborative and innovative approach to data support is unique to the OCDSB and can serve as a valuable model to other school districts. A main objective of this Data Support Model is to build greater capacity at the system- and school-level in supporting data-driven decision making and evidence informed practice. As evidenced by the feedback provided by numerous stakeholders, this model is exhibiting positive impact across a number of areas. Continued enhancement and growth of the Data Support Model would serve to better support systemand school-level objectives which in turn, will positively impact student learning. Specific opportunities for growth are four-fold. The first opportunity is in the differentiation of supports. It will be vital to create and encourage collaborative opportunities within and across super intendencies and schools to meet their varied needs and build further system capacity. Research further corroborates this approach with greater data-driven decision-making occurring when educators share data within and across schools [9].

The second opportunity is in providing ongoing job embedded learning. It will be necessary to provide guidance to school teams throughout the year to offset peak times for support requests and improve the consistency of data support. DSLs will continue presenting data in a non-evaluative manner that fosters open communication and promotes participation and engagement. Establishing a culture 
of data use through direct support to system leaders, administrators, and educators will further grow Data Literacy at these levels [9].

The third opportunity is to improve the visibility of the Data Support Model. As discussed, only 65\% of schools are currently accessing their Data Support Lead and fewer (62\%) are accessing the Data Support Toolkit. Moving forward, considering strategies aimed at promoting greater interest in the model is warranted. Some examples include increased communication with school administrators, greater sharing and involvement among partners, continued resource development, and a greater online presence and availability of tools/resources. Furthermore, it is posited that the continued role DSLs play in modelling best practices and encouraging open discussion about data findings, will empower administrators to model the effective use of data with educators at their school.

Finally, over the last three years feedback from various stakeholders has been collected in an effort to monitor the progress and sustainability of the revised Data Support Model. Moving forward, continued monitoring for impact at the system, school, and student level is warranted in order to encourage reflection on current instructional practices, growth in Data Literacy [9], building capacity and effects on educator/student learning.

\section{Conclusion}

Overall, this collaborative and innovative Data Support Model has been successful in building District capacity in Data Literacy skills. There has been considerable commitment from participants and a continued desire to grow and enhance this model. With further commitment from senior staff, administrators, educators, and DSLs the model will continue to grow and encourage greater collaborative opportunities, guidance to school teams, modelling of best practices, and monitoring impact and the effects on student learning. With time, it is also anticipated that student outcomes will also be positively impacted. This approach is unique to this school district and as such, can serve as a valuable model to other school districts.

\section{References}

[1] Ebbeler, J., Poortman, C.L., Schildkamp, K., \& Pieters, J.M. (2017). The effects of data use intervention on educator's satisfaction and data literacy. Educational Assessment Evaluation and Accountability, 29: 83-105. doi:10.1007/x11092-016-9251-z.

[2] Falk, B., \& Ort, S. (1998). Sitting down to score: teacher learning through assessment. The Phi Delta Kappan, 80(1), 59-64.
[3] Gambell, T. (2004). Teachers working around largescale assessment: reconstructing professionalism and professional development. English Teaching: Practice and Critique, 3(2), 48-73.

[4] Mandinach, E.B. (2012). A perfect time for data use: using data-driven decision making to inform practice. Educational Psychologist, 47(2), 71-85.

[5] Mandinach, E.B., \& Gummer, E.S. (2012). Navigating the landscape of data literacy: it IS complex. Washington, DC/Portland, OR: WestEd/Education Northwest.

[6] Mandinach, E.B., \& Gummer, E.S. (2013). A systemic view of implementing data literacy in educator preparation. Educational Researcher, 42(1), 30-37. doi: 10.3102/0013189X12459803.

[7] Marsh, J.A., \& Farrell, C. (2015). How leaders can support teachers with data-driven decision making: a framework for understanding capacity building. Educational Management Administration \& Leadership, 43(2), 269-289. Doi: 10.1177/1741143214537229.

[8] McNaughton, S., Lai, M., \& Hsaio, S. (2012). Testing the effectiveness of an intervention model based on data use: a replication series across clusters of schools. School Effectiveness and School IMprovement, 23(2), 203-228. Doi: 10.1080/09243453.2011.652126.

[9] Park, V., \& Datnow, A. (2009). Co-constructing distributed leadership: district and school connections in data-driven decision-making, School Leadership and Management, 29(5), 477-494. DOI: $10.1080 / 13632430903162541$

[10] Reeves, T.D., \& Honig, S.L. (2015). A classroom data literacy intervention for pre-service teachers. Teaching and Teacher Education, 50, 90-101.

[11] Wayman, J.C., \& Jimerson, J.B. (2014). Teacher needs for data-related professional learning. Studies in Educational Evaluation, 42, 25-34.

[12] Wayman, J.C., \& Stringfield, S. (2006). Technologysupported involvement of entire faculties in examination of student data for instructional improvement. American Journal of Education, 112 (August), 549-571. 